\title{
POLAR SETS AND CAPACITARY POTENTIALS IN HOMOGENEOUS SPACES
}

\author{
Tord Sjödin \\ University of Umeå, Department of Mathematics and Mathematical Statistics \\ S-901 87 Umeå, Sweden; tord.sjodin@math.umu.se
}

\begin{abstract}
A set $E$ in a space $X$ is called a polar set in $X$, relative to a kernel $k(x, y)$, if there is a nonnegative measure $\sigma$ in $X$ such that the potential $U_{k}^{\sigma}(x)=\infty$ precisely when $x \in E$. Polar sets have been characterized in various classical cases as $G_{\delta}$-sets (countable intersections of open sets) with capacity zero. We characterize polar sets in a homogeneous space $(X, d, \mu)$ for several classes of kernels $k(x, y)$, among them the Riesz $\alpha$-kernels and logarithmic Riesz kernels. The later case seems to be new even in $\mathbf{R}^{n}$.
\end{abstract}

\section{Introduction}

The classical potential theory has its roots in the study of Newtonian potentials and their connection to the Laplace equation, c.f. Kellogg [15]. The theory was generalized and put on a firm mathematical ground in Frostman's thesis [11]. Its later developments can be found in Landkof [17], Doob [8], Adams and Hedberg [1], Armitage and Gardiner [2] and the references contained there. More recently, nonlinear potential theory in a doubling metric space that supports a Poincaré-type inequality is studied at length by A. Björn and J. Björn in [3].

We will consider potential theory in an abstract setting. Our starting point is to combine the potential theory in locally compact spaces developed by Fuglede [10] in the 1960's with the homogeneous spaces of Coifman and Weiss [5] from the early 1970's. A homogeneous space $(X, d, \mu)$ consists of a quasimetric space $(X, d)$ equipped with a nonnegative doubling measure $\mu$ (see Section 2 for the details). By a kernel $k(x, y)$ we mean a nonnegative and lower semicontinous function $k: X \times X \rightarrow$

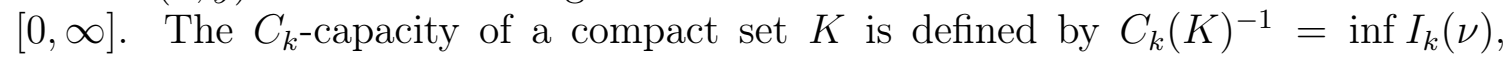
where infimum is over all nonnegative measures $\nu$ supported in $K$ with total mass $\|\nu\|_{1}=1$ and $I_{k}(\nu)$ denotes the energy integral $I_{k}(\nu)=\iint k(x, y) d \nu(x) d \nu(y)$. A complete homogeneous space is locally compact and we can apply the basic existence theorem for capacitary measures and capacitary potentials for compact sets in [10, Theorem 2.4]. The corresponding result for more general sets in a locally compact space is harder and requires stronger assumptions on the kernel, see [10, Chap. II]. The situation is however better if $X$ is a compact space [10, Theorem 3.4.1]. We combine the compact case with a locally finite, open covering of $X$ to prove the existence of a type of capacitary potentials for certain neighbourhoods of sets with capacity zero (Theorem 2.1). Capacitary theorems for Riesz potentials in $\mathbf{R}^{n}$ are proved in [17, Ch. II], and for nonlinear $L^{p}$-potentials in $\mathbf{R}^{n}$ in [1, Ch. 2].

doi:10.5186/aasfm.2013.3851

2010 Mathematics Subject Classification: Primary 26E25; Secondary 46B20, $49 J 50$.

Key words: Metric space, doubling measure, Riesz kernel, definite kernel, consistent kernel, measure, potential, capacity, energy, capacitary measure, capacitary potential, polar set. 
A set $E$ is called a polar set (relative to a kernel $k(x, y)$ ) if there is a nonnegative measure $\nu$ such that the potential $U_{k}^{\nu}(x)$ is infinite precisely when $x \in E$ (see Section 5). Polar sets for the Newton kernel were characterized by Deny [7]. The same proof works for general Riesz kernels, see [17, Ch. III] and the references contained there. Polar sets for superparabolic functions were studied in [16]. Compact polar sets and compact sets with capacity zero were characterized in [22], [13] and [20]. We prove characterizations of polar sets in $(X, d, \mu)$ for a number of classes of kernels, among them the Riesz $\alpha$-kernels and logarithmic Riesz kernels.

It is the purpose of this paper to find classes of kernels in $(X, d, \mu)$, for which we can characterize polar sets in analogy with the classical cases above. We consider kernels $K \circ \mu(x, y)$ adopted to the metric/measure structure of $(X, d, \mu)$, for which there exists an equivalent continuous and definite kernel $k(x, y)$. We find a way to construct such pairs of kernels that, although it follows classical lines, seems to be new. This result is applied to the Riesz $\alpha$-kernels and logarithmic Riesz kernels, the later case seems to be new even in $\mathbf{R}^{n}$. To achieve this, we develop a potential theory for the homogeneous spaces $(X, d, \mu)$ that includes potentials, energies, capacities, capacitary potentials and capacity estimates.

Our main results are stated in Section 2 and proved in Section 6. Section 3 contains our preliminaries on homogeneous spaces, while the basic potential theory in $(X, d, \mu)$ is described in Section 4. Section 5 gives an introduction to potentials with finite energy and contains our construction of the (positive) definite kernels used to characterize polar sets.

\section{Main results}

Our first result is a capacitary theorem for certain neighbourhoods of sets with capacity zero.

Theorem 2.1. Let $(X, d, \mu)$ be a complete homogeneous space of order $\gamma, 0<$ $\gamma<1$, satisfying $(D C)$, let $k(x, y)$ be a continuous and definite kernel and let $K \circ$ $\mu(x, y)$ be a doubling kernel equivalent to $k(x, y)$. Then for every $\epsilon>0$ and every set $E$ with $C_{k}(E)=0$ there is an open set $V$ containing $E$ and $\nu \in M_{+}(\bar{V})$ such that

$$
U_{k}^{\nu}(x) \geq 1, x \in V, \quad U_{k}^{\nu}(x)<\infty, x \in X,
$$

and $\|\nu\|_{1}<\epsilon, C_{k}(V)<\epsilon$.

The next theorem is our principal result, which gives necessary and sufficient conditions for a set $E$ to be a polar set in the sense of Definition 5.1. The proof of statement (a) in Theorem 2.2 is fairly simple, while the proof of (b) requires the full strength of our methods.

Theorem 2.2. Let $(X, d, \mu)$ be a complete homogeneous space of order $\gamma, 0<$ $\gamma<1$, satisfying $(D C)$ and let $K \circ \mu$ be a doubling kernel satisfying (5.6).

(a) If $E$ is a polar set, then $E$ is a $G_{\delta}$-set with $C_{K}(E)=0$.

(b) Let $k(x, y)$ be a continuous and definite kernel equivalent to $K \circ \mu$ and let $E$ be a $G_{\delta^{-}}$set with $C_{K}(E)=0$. Then there is $\sigma \in M_{+}(X)$ such that $U_{K}^{\sigma}(x)=\infty$ precisely when $x \in E$.

Combining statements (a) and (b) in Theorem 2.2 gives the following characterization of polar sets in a homogeneous space and is our main result. 
Theorem 2.3. Let $(X, d, \mu)$ be a complete homogeneous space of order $\gamma, 0<$ $\gamma<1$, satisfying $(D C)$, let $k(x, y)$ be a continuous and definite kernel and let $K \circ \mu$ be a doubling kernel equivalent to $k(x, y)$ and satisfying (5.6). Then a set $E$ is a polar set if only if $E$ is a $G_{\delta^{-}}$set with $C_{K}(E)=0$.

Three special cases of Theorem 2.3 are worth mentioning. The kernel in the first case is the most general kernel that can be handled with our methods, while in the second and third cases the kernels are modeled on the Riesz $\alpha$-kernel in $\mathbf{R}^{n}$ and a Riesz kernel with a logarithmic factor.

Theorem 2.4. Let $(X, d, \mu)$ be a complete homogeneous space of order $\gamma, 0<$ $\gamma<1$, satisfying $(D C)$ and let $K \circ \mu(x, y)$ be a doubling kernel, where $\int_{1}^{\infty} K(t)^{2} d t<$ $\infty$. Define $\tilde{K}(r)$ by (5.2) and assume that $\tilde{K}(r)$ satisfies (4.1). Then a set $E$ is a polar set relative to the kernel $\tilde{K} \circ \mu(x, y)$ if and only if $E$ is a $G_{\delta}$-set with $C_{\tilde{K}}(E)=0$.

Theorem 2.5. Let $(X, d, \mu)$ be a complete homogeneous space of order $\gamma, 0<$ $\gamma<1$, satisfying $(D C)$ and let $K_{\alpha} \circ \mu(x, y), 0<\alpha<1$, be the Riesz kernel. Then a set $E$ is a polar set if and only if $E$ is a $G_{\delta^{-}}$set with $C_{\alpha}(E)=0$.

Theorem 2.6. Let $(X, d, \mu)$ be a complete homogeneous space of order $\gamma, 0<$ $\gamma<1$, satisfying $(D C)$ and let $K_{\alpha, p} \circ \mu(x, y)$, where $0<\alpha<1$ and $p$ is a real number, be the logarithmic Riesz kernel. Then a set $E$ is a polar set if and only if $E$ is a $G_{\delta^{-}}$set with $C_{\alpha, p}(E)=0$.

Remark. By Fubini's Theorem, $\tilde{K}(r)$ satisfies (4.1) iff $\int_{0}^{1} t \cdot K(t)^{2} d t<\infty$ and either $\int_{0}^{1} K(t)^{2} d t=\infty$ or $K(r) \cdot \int_{0}^{r} K(t) d t \rightarrow \infty$, as $r \rightarrow 0+$.

The class of kernels $K \circ \mu(x, y)$ for which polar sets $E$ in $X$ are characterized as $G_{\delta^{-}}$sets with $C_{K}(E)=0$, contains the kernels $\tilde{K} \circ \mu(x, y)$ in Theorem 2.4 and the Riesz $\alpha$-kernels and the logarithmic Riesz kernel in Theorem 2.5 and Theorem 2.6, respectively.

\section{Preliminaries}

We follow [5] and define a homogeneous space as a triple $(X, d, \mu)$, where $(X, d)$ is a quasi-metric space satisfying

$$
d(x, y) \leq K \cdot(d(x, z)+d(z, y)), \quad x, y, z \in X,
$$

for some constant $K \geq 1$, and $\mu$ is a nonnegative and nonatomic measure on the $\sigma$-algebra generated by all balls $B(x, r)=\{y \in X ; d(y, x)<r\}$ and satisfies the doubling condition

$$
0<\mu B(x, 2 r) \leq M \cdot \mu B(x, r), \quad x \in X, r>0,
$$

with doubling constant $M$. It follows from (3.2) that

$$
\mu B(x, r) \geq C \cdot\left(\frac{r}{R}\right)^{\alpha} \cdot \mu B(x, R), \quad x \in X, 0<r<R,
$$

where the constants $C>0$ and $\alpha>0$ only depend on $M$ and $K$. A relation which holds except in a set of $\mu$-measure zero is said to hold $\mu$-a.e. See [5], [6], [14] and [18] for more on the basic properties of homogeneous spaces.

We give $X$ the topology induced by the balls $B(x, r)=\{y \in X ; d(y, x)<r\}$ and denote the closure and complement of a set $E \subset X$ by $\bar{E}$ and $E^{c}$ respectively. Let $\bar{B}(x, r)=\{y \in X ; d(y, x) \leq r\}$ and $S(x, r)=\{y \in X ; d(y, x)=r\}$. It follows from 
[5, Theorem 1.2] that bounded sets are totally bounded. If $(X, d)$ is complete then $(X, d)$ is a locally compact and separable Hausdorff space, see $[19$, Ch. 4 , Sec. 25 , Theorem A]. Every open set is a countable union of balls $B(x, r)$ and thus $\mu$-measurable. Then $\mu$ is a regular Borel measure on $X$.

We say that $(X, d, \mu)$ is of order $\gamma, 0<\gamma<1$, if there is a constant $C$ such that

$$
|d(x, z)-d(z, y)| \leq C \cdot R^{1-\gamma} \cdot d(x, y)^{\gamma},
$$

for all $x, y \in B(z, R), z \in X$ and $R>0$. Every homogeneous space has an equivalent quasinorm satisfying (3.4), c.f. [18, Theorem 2]. In that case all sets $B(x, r)$ and $\{y ; d(y, x)>r\}$ are open sets in $X$. We say that a homogeneous space satisfies a density condition (DC) if there are constants $N \geq 2$ and $A>1$ such that

$$
\mu B(x, r) \geq A \cdot \mu B(x, r / N), \quad x \in X, r>0 . \quad(D C)
$$

The condition (DC) implies that

$$
\mu B(x, r) \leq N^{\beta} \cdot(r / R)^{\beta} \cdot \mu B(x, R), \quad x \in X, 0<r \leq R,
$$

where $\beta$ only depends on $A$ and $N$, in analogy with (3.3). We will also need the following continuity property for $\mu$ on balls $\bar{B}(x, r)$.

Lemma 3.1. Let $(X, d, \mu)$ be a homogeneous space of order $\gamma, 0<\gamma<1$, and assume that

$$
\mu S(x, r)=\mu\{y ; d(x, y)=r\}=0, \quad x \in X, r>0
$$

Then $\mu \bar{B}(x, r)$ and $\mu \bar{B}(x, d(x, y))$ are continuous functions of $x \in X, r \geq 0$ and $x, y \in X$, respectively.

Proof. It is enough to treat $\mu \bar{B}(x, r)$, since $d(x, y)$ is a continuous function of $x, y \in X$. Fix any $x_{0} \in X$ and $r_{0} \geq 0$. Then

$$
\bar{B}(x, r) \subset \bar{B}\left(x_{0}, r_{0}+C \cdot d\left(x, x_{0}\right)^{\gamma}+\left|r-r_{0}\right|\right),
$$

which gives $\lim \sup \mu \bar{B}(x, r) \leq \mu \bar{B}\left(x_{0}, r_{0}\right)$, as $(x, r) \rightarrow\left(x_{0}, r_{0}\right)$. For $r_{0}>0$, an analogous inclusion gives $\mu \bar{B}\left(x_{0}, r_{0}\right) \leq \liminf \mu \bar{B}(x, r)$, as $(x, r) \rightarrow\left(x_{0}, r_{0}\right)$

Our notation for measures and integrals are standard. We let $M(X)$ denote the class of Borel measures $\nu$ on a $X$, with finite mass on bounded sets, and we let $M_{+}(X)$ be the subclass of positive measures. If $E$ is a Borel set $M(E)$ is the class of $\nu \in M(X)$ that are concentrated on $E$ and analogously for $M_{+}(E)$. The closed support and total variation of a measure $\nu$ in $M(X)$ are denoted by $\operatorname{supp}(\nu)$ and $\|\nu\|_{1}$, respectively. Various constants, that may vary from one instance to another, are written $C, C_{1}, C_{2}, \ldots$

\section{Basic potential theory in $(X, d, \mu)$}

We define the basic potential theoretical concepts as in [10, Ch. I.2] or [17, Ch. II]. By a kernel $k(x, y)$ we mean a symmetric, nonnegative and lower semicontinuous function $k: X \times X \rightarrow[0, \infty]$. We define the potential $U_{k}^{\nu}(x)$ (relative to the kernel $k$ ) and the energy $I_{k}(\nu)$ of $\nu \in M_{+}(X)$ by

$$
U_{k}^{\nu}(x)=\int k(x, y) d \nu(y) \quad \text { and } \quad I_{k}(\nu)=\iint k(x, y) d \nu(x) d \nu(y),
$$


respectively. We follow [10] and define a set function $w_{k}(E)=\inf I_{k}(\nu)$, where infimum is over all $\nu \in M_{+}(X),\|\nu\|_{1}=1$, that are concentrated on $E$. The inner capacity $\underline{C}_{k}(E)$ and the outer capacity $\bar{C}_{k}(E)$ of $E$ are then defined by

$$
\underline{C}_{k}(E)=1 / w_{k}(E) \quad \text { and } \quad \bar{C}_{k}(E)=\left(\sup _{E \subset G} w_{k}(G)\right)^{-1},
$$

respectively, where supremum is taken over open sets $G$. As usual, a set $E$ is called capacitable if $\underline{C}_{k}(E)=\bar{C}_{k}(E)$ and this common value is written $C_{k}(E)$. Open sets and compact sets are capacitable and

$$
\underline{C}_{k}(E)=\sup _{K \subset E} C_{k}(K), \quad \bar{C}_{k}(E)=\inf _{E \subset G} C_{k}(G),
$$

where $K$ and $G$ denote compact and open sets respectively [10]. The basic existence theorem for capacitary measures and capacitary potentials for compact sets are proved in [10, Theorem 2.4]. The corresponding results for more general sets require stronger assumptions on the kernel, c.f. [10, Ch. II].

The potential $U_{k}^{\nu}(x)$ of a signed measure $\nu=\nu_{+}-\nu_{-}$in $M(X)$, where $\nu_{+}, \nu_{-} \in$ $M_{+}(X)$, is defined by

$$
U_{k}^{\nu}(x)=\int k(x, y) d \nu_{+}(y)-\int k(x, y) d \nu_{-}(y),
$$

provided at least one of the two integrals is finite. Analogously, we define the mutual energy of two measures $\nu, \tau \in M(X)$ by $I(\nu, \tau)=\int U_{k}^{\nu}(x) d \tau(x)$, whenever the integral is well defined, and put $I(\nu)=I(\nu, \nu)$. A kernel $k(x, y)$ is called a (positive) definite kernel if $I(\nu) \geq 0$ for all $\nu \in M(X)$ such that $I(\nu)$ is defined. Such kernels are studied at length in [10, Ch. II]. We will return to this case in the next section.

A relation which holds except in a set of $C_{k}$-capacity zero is said to hold $C_{k}$-quasi everywhere, written $C_{k}$-q.e., and analogously for $\underline{C}_{k}$ and $\bar{C}_{k}$. Clearly, $C_{k}(K)>0$ if and only if there exists a nonzero measure $\nu \in M_{+}(K)$ with finite energy $I_{k}(\nu)$.

A kernel $k(x, y)$ is continuous if it is finite and continuous for $x \neq y$. We say that $k(x, y)$ is doubling if $d(x, z) \leq 2 K \cdot d(x, y)$ implies that $k(x, y) \leq C \cdot k(x, z)$, where $C$ is independent of $x, y, z \in X$. Two kernels $k_{1}(x, y)$ and $k_{2}(x, y)$ are equivalent if $C$. $k_{1}(x, y) \leq k_{2}(x, y) \leq k_{1}(x, y) / C$, for some positive constant $C$ independent of $x, y \in$ $X$. Clearly, equivalent kernels have equivalent potentials, energies and capacities. In the following we consider kernels adopted to the metric/measure structure of $(X, d, \mu)$ defined by

$$
K \circ \mu(x, y)=K(\mu \bar{B}(x, d(x, y))+\mu \bar{B}(y, d(x, y))),
$$

where $K:(0, \infty) \rightarrow[0, \infty)$ is nonincreasing and continuous, see [21]. Then $K \circ \mu$ has the properties of a kernel above, provided $\mu$ is of order $\gamma$ for some $0<\gamma<1$. If $\mu$ also satisfies (3.6), then $K \circ \mu(x, y)$ is continuous by Lemma 3.1. We also assume that $K(r)$ satisfies the doubling condition $K(r) \leq B \cdot K(2 r), r>0$, for some $B>1$, then $K \circ \mu$ is doubling in the sense defined above. Moreover, we assume that $K(r)$ satisfies the standard conditions

$$
\lim _{r \rightarrow 0+} K(r)=\infty \quad \text { and } \quad \int_{0}^{1} K(r) d r<\infty .
$$

Remark. Continuity properties of potentials for kernels $K(d(x, y))$ depending on the distance are studied in [12]. As in [21], we can use the doubling measure $\mu$ to define another quasi distance $d_{1}$ on $X$ by $d_{1}(x, y)=\inf \mu(B)$, where infimum is over 
all open balls $B$ containing $x$ and $y$. Then $K \circ \mu(x, y) \sim K\left(d_{1}(x, y)\right)$, provided $K(r)$ is doubling, and our case relates to [12].

Potentials, capacities and energies relative to a kernel $K \circ \mu$ are denoted by $U_{K}^{\nu}$, $C_{K}$ and $I_{K}$, respectively. Then $C_{K}(\{x\})=0, x \in X$, since $I_{K}\left(\nu_{x}\right)=\infty$ for the Dirac measure $\nu_{x}$ at $x$. We want to compare the capacity $C_{K}$ and the measure $\mu$ of balls $\bar{B}(x, r)$ using the following lemma.

Lemma 4.1. Let $(X, d, \mu)$ be a homogeneous space satisfying $(D C)$ and let $K \circ \mu$ be a doubling kernel. Then

$$
\int_{d(y, x) \leq r} K \circ \mu(x, y) d \mu(y) \sim \int_{0}^{\mu \bar{B}(x, r)} K(t) d t, \quad x \in X, r>0,
$$

and

$$
\int_{d(y, x) \geq r} K \circ \mu(x, y) d \mu(y) \sim \int_{\mu \bar{B}(x, r)}^{\infty} K(t) d t, \quad x \in X, r>0,
$$

with constants depending on the doubling constants for $\mu$ and $K(r)$ and the numbers $A, N$ in $(D C)$.

Proof. Fix any $x \in X, r>0$, let $N \geq 2$ be the constant in (DC) and define circular sets

$$
E_{\nu}=\left\{y ; N^{-\nu} \cdot r<d(y, x) \leq N^{1-\nu} \cdot r\right\}, \quad \nu \text { integer. }
$$

Then $\mu E_{\nu} \sim \mu \bar{B}\left(x, N^{1-\nu} r\right)$ and for the integral in the left hand side of (4.2) we get

$$
\sum_{\nu=1}^{\infty} \int_{E_{\nu}} K \circ \mu(x, y) d \mu(y) \sim \sum_{\nu=1}^{\infty} K\left(\mu \bar{B}\left(x, N^{-\nu} \cdot r\right)\right) \cdot \mu \bar{B}\left(x, N^{1-\nu} \cdot r\right) .
$$

Put $b_{\nu}=\mu \bar{B}\left(x, N^{-\nu} \cdot r\right)$, then for the integral on the right hand side of (4.2) we have

$$
\begin{aligned}
\sum_{\nu=1}^{\infty} \int_{b_{\nu}}^{b_{\nu-1}} K(t) d t & \left.\sim \sum_{\nu=1}^{\infty} K\left(\mu \bar{B}\left(x, N^{1-\nu} \cdot r\right)\right) \cdot\left(\mu \bar{B}\left(x, N^{1-\nu} \cdot r\right)\right)-\mu \bar{B}\left(x, N^{-\nu} \cdot r\right)\right) \\
& \sim \sum_{\nu=1}^{\infty} K\left(\mu \bar{B}\left(x, N^{-\nu} \cdot r\right)\right) \cdot \mu \bar{B}\left(x, N^{1-\nu} \cdot r\right)
\end{aligned}
$$

by (DC), with constants depending on $\mu$ and $K(r)$. The relation (4.3) is proved in the same way.

The following lower bound for $C_{K}(B(x, r))$ will be used in the proof of Theorem 2.2(b), c.f. [1, Ch. 5].

Lemma 4.2. Let $(X, d, \mu)$ be a homogeneous space satisfying $(D C)$ and let $K \circ \mu$ be a doubling kernel. Then

$$
C_{K}(\bar{B}(x, r)) \geq C \cdot \mu \bar{B}(x, r) \cdot\left(\int_{0}^{\mu \bar{B}(x, r)} K(t) d t\right)^{-1}, x \in X, r>0,
$$

where $C$ only depends on the doubling constants for $\mu$ and $K(r)$ and the numbers $A, N$ in (DC). Moreover, if $K$ is any compact set with $C_{K}(K)=0$, then $\mu(K)=0$.

Proof. Let $B=\bar{B}\left(x_{0}, r_{0}\right), r_{0}>0$ and $\nu=\mu(B)^{-1} \cdot \mu_{0}$, where $\mu_{0}$ is the restriction of $\mu$ to $B$. Then $\nu \in M_{+}(B),\|\nu\|_{1}=1$ and $C_{K}(B) \geq I_{K}(\nu)^{-1}$. In the following we find 
upper estimates for $U_{K}^{\mu_{0}}(x)$ and $I_{K}\left(\mu_{0}\right)$ and start with $U_{K}^{\mu_{0}}(x)$. If $d\left(x, x_{0}\right) \leq 4 K^{2} r_{0}$, then $d(x, y) \leq 5 K^{3} r_{0}$ for all $y \in B$ and

$U_{K}^{\mu_{0}}(x) \leq \int_{d(y, x) \leq 5 K^{3} r_{0}} K \circ \mu(x, y) d \mu(y) \leq C \cdot \int_{0}^{\mu \bar{B}\left(x, 5 K^{3} r_{0}\right)} K(t) d t \leq C \cdot \int_{0}^{\mu(B)} K(t) d t$

by Lemma 4.1 and the doubling properties of $\mu$ and $K(r)$. If $d\left(x, x_{0}\right) \geq 4 K^{2} r_{0}$ then $B \subset \bar{B}(y, d(x, y))$ for all $y \in B$ and

$$
U_{K}^{\mu_{0}}(x) \leq K(\mu(B)) \cdot \mu(B) \leq \int_{0}^{\mu(B)} K(t) d t .
$$

Hence $I_{K}\left(\mu_{0}\right) \leq C \cdot \mu(B) \cdot \int_{0}^{\mu(B)} K(t) d t$ and (4.2) follows from the definition of $\nu$. Finally, let $K \subset \bar{B}\left(x_{0}, r_{0}\right)$ be a compact set with $\mu(K)>0$ and let $\mu_{1}$ be the restriction of $\mu$ to $K$, then $I_{K}\left(\mu_{1}\right) \leq I_{K}\left(\mu_{0}\right)<\infty$ and therefore $C_{K}(K)>0$. This completes the proof of Lemma 4.2.

\section{Potentials with finite energy}

Potentials of definite kernels have played an important role in the development of modern potential theory, see [10, Ch. II.3]. Recall that a kernel $k(x, y)$ is called definite if $I_{k}(\nu) \geq 0$ for all $\nu \in M(X)$ such that $I_{k}(\nu)$ is defined. Let $\mathcal{E}(X)$ be the class of $\nu \in M(X)$ such that $I_{k}(\nu)$ is well defined, nonnegative and finite. Then $\mathcal{E}(X)$ is a pre-Hilbert space with scalar product $I(\nu, \tau)$ and norm $\|\nu\|=I(\nu)^{1 / 2}$, $\nu, \tau \in \mathcal{E}(X)$. The subspace of $\mathcal{E}(X)$ of nonnegative measures is denoted by $\mathcal{E}_{+}(X)$. Convergence in the seminorm $\|\cdot\|$ is called strong convergence in $\mathcal{E}(X)$.

We show that some of the kernels $K \circ \mu$ have equivalent definite kernels. Let $k(x, y)$ be a kernel (not necessarily of the form $K \circ \mu$ ) and let $\nu \in M(X)$ be a measure such that $U_{k}^{\nu}(z)$ is well defined $\mu$-a.e., then

$$
0 \leq \int d \mu(z) U_{k}^{\nu}(z)^{2}=\int d \nu(x) \int d \nu(y) \int k(x, z) k(z, y) d \mu(z)=I_{k_{1}}(\nu),
$$

by Fubini's theorem, where $k_{1}(x, y)=\int k(x, z) k(z, y) d \mu(z)$. It is easy to see that $k_{1}(x, y)$ is a, possibly infinite, definite kernel. The following lemma gives an equivalent form of $k_{1}(x, y)$, when $k(x, y)=K \circ \mu(x, y)$.

Lemma 5.1. Let $(X, d, \mu)$ be a homogeneous space, where $\mu$ satisfies $(D C)$, and let $K \circ \mu$ be a doubling kernel, then

$$
K_{1}(x, y) \stackrel{\text { def }}{=} \int K \circ \mu(x, z) \cdot K \circ \mu(z, y) d \mu(z) \sim \tilde{K} \circ \mu(x, y),
$$

where

$$
\tilde{K}(r)=K(r) \cdot \int_{0}^{r} K(t) d t+\int_{r}^{\infty} K(t)^{2} d t
$$

and the constants only depend on $\mu$ and $K(r)$.

Proof. Fix any $x \neq y$ in $X$ and let $d(x, y)=d$. We split the integral in the left hand side of (5.1) over sets $E_{1}=\{z ; d(z, x)<d / 2 K\}, E_{2}=\{z ; d(z, y)<d / 2 K\}$ and $E_{3}=\{z ; d(z, x) \geq d / 2 K$ and $d(z, y) \geq d / 2 K\}$. The integrals over $E_{1}$ and $E_{2}$ are equivalent to the first term in (5.2), while the integral over $E_{3}$ is equivalent to the second term, by Lemma 4.1 . 
For any doubling kernel $K \circ \mu(x, y)$ we define $K_{1}(x, y)$ and $\tilde{K} \circ \mu(x, y)$ by $(5.1)$ and (5.2), where we assume that $\int_{1}^{\infty} K(t)^{2} d t<\infty$. Clearly, $\tilde{K}(r)$ is continuous, doubling and nonincreasing, since $K(r)$ is absolutely continuous and $\tilde{K}^{\prime}(r)=$ $K^{\prime}(r) \int_{0}^{r} K(t) d t \leq 0$ a.e. Then $K_{1}(x, y)$ is a definite and doubling kernel equivalent to $\tilde{K} \circ \mu(x, y)$.

Next we consider the special choice of $K_{\alpha}(r)=r^{-\alpha}, 0<\alpha<1$, and define the Riesz kernel $K_{\alpha} \circ \mu(x, y)=(\mu \bar{B}(x, d)+\mu \bar{B}(y, d))^{-\alpha}$, where $d=d(x, y)$, and

$$
k_{\alpha}(x, y)=\int K_{\beta} \circ \mu(x, z) \cdot K_{\beta} \circ \mu(z, y) d \mu(z),
$$

with $\beta=(1+\alpha) / 2$. The $k_{\alpha}(x, y)$ is a definite kernel and $K_{\alpha} \circ \mu(x, y) \sim k_{\alpha}(x, y)$, with constants depending on $\mu$ and $\alpha$, by Lemma 5.1 and a straight forward calculation.

More generally, let $1 / 2<\beta<1$, let $p$ be a real number and put $K_{\beta, p}(r)=$ $(\log (1 / r))^{p} \cdot r^{-\beta}, 0<r<r_{0}$, and $K_{\beta, p}(r)=\left(\log \left(1 / r_{0}\right)\right)^{p} \cdot r^{-\beta}, r>r_{0}$, where $r_{0}=\min \left(e^{p / \beta}, 1 / 2\right)$. Then $K_{\beta, p}(r)$ is continuous, decreasing and doubling. Define $k_{\alpha, 2 p}(x, y)$ by $(5.3)$ with $K_{\beta}(r)$ replaced by $K_{\beta, p}(r)$. Then $k_{\alpha, 2 p}(x, y)$ is a definite kernel. Standard calculations show that $\tilde{K}_{\beta, p}(r) \sim K_{\alpha, 2 p}(r)$, where $\tilde{K}_{\beta, p}(r)$ is given by (5.2) and hence $k_{\alpha, 2 p}(x, y) \sim K_{\alpha, 2 p} \circ \mu(x, y)$. We denote the capacity relative to $K_{\alpha, p} \circ \mu$ by $C_{\alpha, p}$.

We will need one more property of our kernels: A kernel $k(x, y)$ satisfies the continuity principle if, whenever a potential $U_{k}^{\nu}(x)$ is continuous as a function on the closed support of $\nu$, then it is continuous in the entire space c.f. [10, p. 150].

Lemma 5.2. Let $(X, d, \mu)$ be a complete homogeneous space of order $\gamma, 0<$ $\gamma<1$. Then any continuous and doubling kernel $K \circ \mu(x, y)$ satisfies the continuity principle.

Remark. In the terminology of [10], a kernel that satisfies the continuity principle is called a regular kernel.

Proof of Lemma 5.2. Let $\nu \in M_{+}(X)$ and assume that $F=\operatorname{supp} \nu$ is compact. It is sufficient to prove that $U_{K}^{\nu}(x)$ is continuous on $F$. Let $\epsilon>0$, then by the uniform continuity of $U_{k}^{\nu}(x)$ on $F$, there is $\delta>0$ such that $u_{2 K \delta}(x)<\epsilon$ for all $x \in F$, where $u_{\delta}(x)$ is the integral $\int K \circ \mu(x, y) d \nu(y)$ over the set $\{y ; d(y, x)<\delta\}$. Let $x$ be any point in $X$. If $d(x, F) \geq \delta$, then $u_{\delta}(x)=0$. If $d(x, F)<\delta$ we let $z$ be a point in $F$ closest to $x$. Then

$$
u_{\delta}(x)=\int_{d(y, x)<\delta} K \circ \mu(x, y) d \nu(y) \leq C \cdot \int_{d(y, z)<2 K \delta} K \circ \mu(z, y) d \nu(y)<C \cdot \epsilon
$$

by the doubling property of $K \circ \mu(x, y)$, which proves that $U_{K}^{\nu}(x)$ is continuous on $X$ in this case. If $F$ is unbounded put $F_{R}=F \cap \bar{B}\left(x_{0}, R\right)$, for some $x_{0} \in F$, and let $\nu_{R}$ be the restriction of $\nu$ to $F_{R}$. Then $U_{K}^{\nu_{R}}(x) \rightarrow U_{K}^{\nu}(x)$, as $R \rightarrow \infty$, uniformly in neighbourhoods of $x_{0}$, by the doubling property of $K \circ \mu(x, y)$, which completes the proof of Theorem 5.2.

A definite kernel $k(x, y)$ is called consistent if every strong Cauchy filter in $\mathcal{E}_{+}(X)$ with a weak limit point $\nu \in M_{+}(X)$ converges strongly to $\nu$ [10, p. 167]. If $k(x, y)$ is a consistent kernel on a locally compact space, then every set $E$ with $\underline{C}_{k}(E)<\infty$ has a capacitary measure and a capacitary potential [10, Theorem 4.1]. In particular, this holds for open sets $G$ with $C_{k}(G)<\infty$. If the space is compact it is sufficient 
that $k(x, y)$ is definite and satisfies the continuity principle by [10, Theorem 3.4.1]. We state this fact in a form that is suitable for our purposes.

Lemma 5.3. Let $(X, d, \mu)$ be a complete homogeneous space of order $\gamma, 0<$ $\gamma<1$, and let $k(x, y)$ be a continuous, doubling and definite kernel. Then for every bounded open set $G$ there is $\nu \in M_{+}(\bar{G})$ such that $\|\nu\|_{1}=1$ and

$$
\begin{array}{ll}
U_{k}^{\nu}(x) \geq 1 / C_{k}(\bar{G}), & \underline{C}_{k} \text {-q.e. } x \in G, \\
U_{k}^{\nu}(x) \leq 1 / C_{k}(\bar{G}), & x \in \operatorname{supp} \nu .
\end{array}
$$

Proof. Let $B=\bar{B}(a, r)$ be a closed ball containing $G$, then $B$ is a compact Hausdorff space with the topology from $X$. Let $k_{0}(x, y)$ be the restriction of $k(x, y)$ to $B$. Then $k_{0}(x, y)$ is a definite kernel on $B$ and $C_{k_{0}}(K)=C_{k}(K)$ for all compact subsets $K$ of $B$, since $I_{k_{0}}(\nu)=I_{k}(\nu)$ for $\nu \in M_{+}(B)$. Moreover, $k_{0}(x, y)$ satisfies the continuity principle in Lemma 5.2 and hence $k_{0}(x, y)$ is a consistent kernel on $B$, by [10, Theorem 3.4.1]. Then by [10, Theorem 4.1] there is $\nu \in M_{+}(\bar{G}),\|\nu\|_{1}=1$ such that (5.3) and (5.4) hold with $U_{k_{0}}^{\nu}(x)$ instead of $U_{k}^{\nu}(x)$. The lemma follows, since these potentials agree on the compact set $B$.

In the classical case the capacitary potential $U_{k}^{\nu}(x)$ in Lemma 5.3 satisfies (5.4) everywhere in $G$, c.f. [17, Ch. II]. A slightly weaker result holds in our case.

Lemma 5.4. Let $(X, d, \mu), k(x, y)$ and $G$ be as in Lemma 5.3 and let $K \circ \mu(x, y)$ be a doubling kernel equivalent to $k(x, y)$. Then there are positive numbers $m$ and $M$, only depending on $\mu$ and the equivalence between the kernels, such that $U_{k}^{\nu}(x) \geq$ $m / C_{k}(\bar{G}), x \in G$, and $U_{k}^{\nu}(x) \leq M / C_{k}(\bar{G}), x \in X$.

Proof. For the sake of simplicity, we let $k(x, y)$ denote any of the kernels $k(x, y)$ and $K \circ \mu(x, y)$. The second inequality is a standard maximum principle for doubling kernels, c.f. [17, Ch. I]. We prove the first inequality with an idea from [4, Sec. III]. The exceptional set in (5.4) is a Borel set $E$ such that $C_{k}(K)=0$ and hence $\mu(K)=0$, for all compact subsets of $E$. Thus (5.4) holds $\mu$-a.e. by the regularity of $\mu$. Fix any $x \in G$ and define

$$
q(z, x)=\frac{k(z, x)}{\int_{0<d(w, x)<d(z, x)} k(w, x) d \mu(w)},
$$

for $0<a<d(z, x)<b$, and $q(z, x)=0$ elsewhere, c.f. [4, Sec. III]. For every $b>0$ there is $0<a<b$ such that $1 \leq \int q(z, x) d \mu(z) \leq C$, where $C$ only depends on $\mu$ and $K(r)$. To see this we note that $\int q(z, x) d \mu(z)>1$, if $a$ is small enough, and $\int q(z, x) d \mu(z) \leq C$, if $a=b / N$ and $N$ is the number in (DC).

Assume that $B(x, b) \subset G$, then we get

$$
C_{k}(\bar{G})^{-1} \leq \int U_{k}^{\nu}(z) \cdot q(z, x) d \mu(z)=\int d \nu(y) \int k(z, y) \cdot q(z, x) d \mu(z) .
$$

Denote the inner integral by $I(x, y)$. It is enough to show that $I(x, y) \leq C \cdot k(x, y)$. Fix any $y \neq x$. If $d(z, x) \leq 2 K d(z, y)$ then $k(z, y) \leq C \cdot k(x, y)$ by the doubling property of $K(r)$. Otherwise, $d(z, y)<d(z, x) / 2 K$ which implies that $d(x, y) / 2 K \leq$ $d(z, x) \leq 2 K d(x, y)$ and $I(x, y) \leq C \cdot k(x, y)$, by Lemma 4.1.

We conclude this section with a lemma on the definition of polar sets. In the study of sets where potentials $U_{k}^{\nu}(x)$ are infinite, it is natural to exclude the case 
where the potentials are identically infinite. We do this in the next lemma. Then we can define polar sets in $(X, d, \mu)$ as in [17, Ch. III].

Lemma 5.5. Let $(X, d, \mu)$ be a complete homogeneous space and let $K \circ \mu$ be a doubling kernel.

(a) If $\nu \in M_{+}(X)$ satisfies

$$
\int_{d(x, a)>1} K(\mu \bar{B}(a, d(x, a)) d \nu(x)<\infty
$$

for some $a \in X$, then $U_{K}^{\nu}(x)<\infty$, for $C_{K}$-q.e. $x \in X$.

(b) If $\nu \in M_{+}(X)$ and (5.6) does not hold, then $U_{K}^{\nu}(x)=\infty$ for all $x \in X$.

Definition 5.1. A set $E$ is called a polar set relative to a doubling kernel $K \circ$ $\mu(x, y)$ if there is $\nu \in M_{+}(X)$ satisfying (5.6) such that $U_{K}^{\nu}(x)=\infty$ precisely when $x \in E$.

Remark. The condition (5.6) is clearly independent of the choice of the point $a$, by the doubling property of $\mu$ and $K(r)$.

Proof of Lemma 5.5. To prove (a) we put $E=\left\{x ; U_{K}^{\nu}(x)=\infty\right\}$. Let $B(a, r)$ be a fixed ball, define $E_{r}=E \bigcap B(a, r)$ and let $\nu_{r}$ be the restriction of $\nu$ to $B(a, 2 r)$. Then for $x \in B(a, r)$ we have $U_{K}^{\nu}(x)=\infty$ if and only if $U_{K}^{\nu_{r}}(x)=\infty$. By [10, Lemma 2.3.5] it is sufficient to prove that $\bar{C}_{K}\left(E_{r}\right)=0$ for all $r>0$. Let $K$ be a compact subset of the open set $G_{n}=\left\{x ; U_{K}^{\nu_{r}}(x)>n\right\}$ and let $\tau$ be the capacitary measure for $K$, then

$$
n<\int U_{K}^{\nu_{r}}(x) d \tau(x)=\int U_{K}^{\tau}(x) d \nu_{r}\left(x \leq C \cdot\left\|\nu_{r}\right\|_{1} / C_{K}(K) .\right.
$$

Hence $C_{K}\left(G_{n}\right) \leq C \cdot\|\nu\|_{1} / n$ and $C_{K}\left(E_{r}\right)=0$ by the outer regularity of $C_{K}$. Finally, if (5.6) fails, then for any fixed $x \in X$

$$
U_{K}^{\nu}(x) \geq C \cdot \int_{d(y, x) \geq 1} K(\mu \bar{B}(x, d(x, y))) d \nu(y)=\infty,
$$

which proves (b).

\section{Proofs}

We begin with the proof of Theorem 2.1 and construct the desired open neighbourhood $G$ of $E$ as $G=\bigcup_{1}^{\infty} G_{n}$, where $\left\{G_{n}\right\}_{1}^{\infty}$ is a locally finite, open covering of $E$ such that $C_{k}(G) \leq \sum_{1}^{\infty} C_{k}\left(G_{n}\right)$ is small.

Proof of Theorem 2.1. Let $\epsilon>0$ be arbitrary and let $\left\{a_{n}\right\}_{1}^{\infty}$ be positive numbers such that $\sum_{1}^{\infty} a_{n}<m \cdot \epsilon$, where $m$ is the number in Lemma 5.4. Fix $a \in X$ and define open sets $V_{1}=\{x ; d(x, a)<2 K\}$ and $V_{n}=\left\{x ;(2 K)^{n-2}<d(x, a)<(2 K)^{n-1}\right\}$, $n \geq 2$, where $K$ is the constant in (3.1). Then $\left\{V_{n}\right\}_{1}^{\infty}$ is a locally finite, open covering of $X$, such that every $x \in X$ belongs to at most two sets $V_{n}$. Further, the distance between $V_{n}$ and $V_{p}$ is at least $(2 K)^{n+1}$, for $p \geq n+3$. Choose open sets $G_{n} \subset V_{n}$ such that $E \bigcap V_{n} \subset G_{n}$ and $C_{k}\left(G_{n}\right)<a_{n}$. Let $\nu_{n}$ be the capacitary measure for $G_{n}$ from Lemma 5.3. Define $\sigma_{n}=C_{k}\left(G_{n}\right) / m \cdot \nu_{n}$, then $U_{k}^{\nu_{n}}(x) \geq 1, x \in G_{n}$, by Lemma 5.4, and $\|\sigma\|_{1}<a_{n} / m, n \geq 1$. Put $\sigma=\sum_{1}^{\infty} \sigma_{n}$ and $G=\bigcup_{1}^{\infty} G_{n}$, then 
$\|\sigma\|_{1} \leq \sum_{1}^{\infty} a_{n} / m<\epsilon, C_{k}(G) \leq \sum_{1}^{\infty} C_{k}\left(G_{n}\right)<\epsilon$ and $U_{k}^{\sigma}(x) \geq 1, x \in G$. Finally, let $x \in V_{n}$ for some fixed $n$, then

$$
U_{k}^{\sigma}(x)=\sum_{|p-n| \leq 2} U_{k}^{\sigma_{p}}(x)+\sum_{|p-n|>2} U_{k}^{\sigma_{p}}(x)=I+I I .
$$

If $|p-n|>2$, then $d(x, y) \geq(2 K)^{2}$ for all $y$ in the support of $\sigma_{p}$ and $U_{k}^{\sigma_{p}}(x) \leq$ $K\left(\mu \bar{B}\left(x,(2 K)^{2}\right)\right) \cdot\left\|\sigma_{p}\right\|_{1}$, which proves that $I I$ is finite. Clearly, $I$ is finite by Lemma 5.4 and the proof of Theorem 2.1 is complete.

The proof of part (a) in Theorem 2.2 is fairly standard, while the proof of (b) uses the capacitary measure in Theorem 2.1 and the estimate for the capacity of a ball $\bar{B}(x, r)$ in Lemma 4.2 .

Proof of Theorem 2.2. The proof of (a) follows from the lower semicontinuity of the potential and Lemma 5.5(a). In the following we assume that $E$ is a $G_{\delta^{-}}$ set with $C_{k}(E)=0$. Recall the open sets $\left\{V_{n}\right\}_{1}^{\infty}$ from the proof of Theorem 2.1. Let $\left(a_{n}\right)_{1}^{\infty}$ and $\left(b_{p}\right)_{1}^{\infty}$ be decreasing sequences of positive numbers, to be specified below, and choose open sets $G_{n}, n \geq 1$, such that $E=\bigcap_{1}^{\infty} G_{n}, C_{k}\left(G_{n}\right)<a_{n}$ and $C_{k}\left(G_{n} \cap V_{p}\right)<a_{n} \cdot b_{p+1}, n, p \geq 1$. For fixed $n \geq 1$, define closed sets

$$
f_{n, m}=\left\{x \in G_{n} ;(2 K)^{-m-1} \leq d\left(x, \partial F_{n}\right) \leq(2 K)^{-m}\right\}, \quad m \in \mathbf{Z},
$$

where $F_{n}=X \backslash G_{n}$. Then $G_{n}=\bigcup_{m} f_{n, m}, n \geq 1$. We claim that $f_{n, m}=\phi$, for $m<n$, provided only the numbers $\left(a_{n}\right)_{1}^{\infty}$ and $\left(b_{p}\right)_{1}^{\infty}$ are choosen small enough. Assume that $x_{0} \in f_{n . m}$ and $m<n$, then

$$
\bar{B}\left(x_{0},(2 K)^{-n-1}\right) \subset \bar{B}\left(x_{0},(2 K)^{-m-2}\right) \subset f_{n, m-1} \bigcup f_{n, m} \bigcup f_{n, m+1} \subset G_{n}
$$

by a straight forward calculation. Assume that $x_{0} \in V_{p}, p \geq 2$, then

$$
\bar{B}\left(x_{0},(2 K)^{-n-1}\right) \subset G_{n} \bigcap\left(V_{p-1} \bigcup V_{p} \bigcup V_{p+1}\right)
$$

and $C_{k}\left(\bar{B}\left(x_{0},(2 K)^{-n-1}\right)\right) \leq 3 \cdot a_{n} \cdot b_{p}$. To get a contradiction we find a lower estimate for the capacity of $B_{0}=\bar{B}\left(x_{0},(2 K)^{-n-1}\right)$. Lemma 4.2 and the doubling properties of $\mu$ and $K(r)$ give

$$
C_{k}\left(B_{0}\right) \geq C / K\left(\mu B_{0}\right) \geq C \cdot(2 K)^{-\alpha n} / K\left(\mu \bar{B}\left(x_{0}, 1\right)\right) \geq C \cdot(2 K)^{-\alpha n} / K\left(m_{p}\right),
$$

where $m_{p}=\inf \left\{\mu \bar{B}(x, 1) ; x \in V_{p}\right\}$, and $\alpha>0$ depends on $\mu$ and $K(r)$. Choosing $a_{n} \leq(2 K)^{-\alpha n}$ and $b_{p}<<K\left(m_{p}\right)^{-1}$ gives the contradiction that proves our claim.

Define sets $E_{n . m}=E \bigcap f_{n, m}$ and choose open sets $G_{n, m}$ such that $E_{n, m} \subset G_{n, m}$ and

$$
C_{k}\left(G_{n \cdot m}\right)<a_{m}, \quad G_{n, m} \subset f_{n, m-1} \bigcup f_{n, m} \bigcup f_{n, m+1}, \quad m \geq n \geq 1 .
$$

Let $\sigma_{n, m}$ denote the capacitary measure constructed for $G_{n, m}$ in Theorem 2.1 such that $U_{k}^{\sigma_{n . m}} \geq 1, x \in G_{n, m}$, and $\left\|\sigma_{n, m}\right\|_{1}<a_{m}$. Put

$$
\sigma=\sum_{n=1}^{\infty} \sum_{m \geq n} \sigma_{n, m} .
$$

We claim that $\sigma \in M_{+}(X)$ and $U_{k}^{\sigma}(x)=\infty$ precisely when $x \in E$. The first part of the claim follows from the definition of $\sigma$ and the fact that $\|\sigma\|_{1} \leq \sum_{n=1}^{\infty} \sum_{m \geq n} a_{m}<$ $\infty$, by the choice of $\left(a_{n}\right)_{1}^{\infty}$ above. If $x \in E$ then $x$ belongs to some $E_{n, m} \subset G_{n, m}$, 
$m \geq n$, for every $n \geq 1$, and hence $U_{k}^{\sigma}(x)=\infty$ by the definition of $\sigma_{n, m}$. If $x \notin E$ there is $n_{0}=n_{0}(x)$ such that $x \in F_{n}, n>n_{0}$. We split $U_{k}^{\sigma}(x)$ as follows

$$
U_{k}^{\sigma}(x)=\sum_{n \leq n_{0}} \sum_{m \geq n} U_{k}^{\sigma_{n, m}}(x)+\sum_{n>n_{0}} \sum_{m \geq n} U_{k}^{\sigma_{n, m}}(x)=I+I I
$$

and start with $I I$.

Fix any $n>n_{0}$ and $x \in F_{n}$ and suppose that $y$ belongs to the closed support of $\nu$. Let $y \in G_{n, m} \subset f_{n, m-1} \bigcup f_{n, m} \bigcup f_{n, m+1}$. An easy calculation shows that $d(y, x) \geq$ $(2 K)^{-m-1}$. Then

$$
K \circ \mu(x, y) \leq K\left(\mu \bar{B}\left(x,(2 K)^{-m-1}\right)\right) \leq C \cdot(2 K)^{\alpha(m+1)} \cdot K(\mu \bar{B}(x, 1))
$$

by the doubling properties of $\mu$ and $K(r)$. Hence

$$
I I \leq C \cdot K(\mu \bar{B}(x, 1)) \cdot \sum_{n>n_{0}} \sum_{m \geq n}(2 K)^{\alpha(m+1)} \cdot a_{m}<\infty
$$

if the numbers $\left(a_{n}\right)_{1}^{\infty}$ are choosen small enough. To estimate $I$ we fix $1 \leq n \leq n_{0}$. Then $x$ belongs to $\bigcup_{k=m-2}^{k=m+2} f_{n, k}$ for at most a finite number of values of $m$. For all other values of $m$, the distance between $x$ and the closed support of $\nu_{n, m}$ is at least $(2 K)^{-m-4}$, since $\operatorname{dist}\left(f_{n, m}, f_{n, m+2}\right) \geq(2 K)^{-m-2}$ for all $m \in \mathbf{Z}$. Thus we can apply the estimate for $I I$. It follows that $U_{K}^{\nu}(x)$ is finite, which completes the proof of Theorem 2.2.

Remark. The open sets $G_{n}$ in the proof of Theorem 2.2 can be constructed in the following way. Choose open sets $H_{n}$ and $H_{n, p}$ such that $E \subset H_{n}, C_{k}\left(H_{n}\right)<a_{n}$ and $E \bigcap V_{p} \subset H_{n, p}, C_{k}\left(H_{n, p}\right)<a_{n} \cdot b_{p+1} / 3$. Then define $G_{n}=H_{n} \bigcap\left(\bigcup_{p=1}^{\infty} H_{n, p}\right)$. Clearly, $E \subset G_{n}$ and $C_{k}\left(G_{n}\right)<a_{n}$. For fixed $p, G_{n} \cap V_{p}$ is a subset of the union of $H_{n, p-1}, H_{n, p}$ and $H_{n, p+1}$. Hence $C_{k}\left(G \bigcap V_{p}\right)<a_{n} \cdot b_{p}$ and the open sets $\left\{G_{n}\right\}_{1}^{\infty}$ have the desired properties.

Proof of Theorem 2.4. The sufficiency part of the proof follows from Theorem 2.2(a). For the necessity part we note that Lemma 5.4 holds for the definite kernel $K_{1}(x, y)$ defined by (5.1) and therefore also for the equivalent kernel $\tilde{K} \circ \mu(x, y)$. Hence Theorem 2.1 holds for $\tilde{K} \circ \mu(x, y)$ and we can apply the proof of Theorem 2.2(b).

Proof of Theorem 2.5. The proof of Theorem 2.5 is, word by word, the same as the proof of Theorem 2.4, if we replace $K_{1}(x, y)$ and $\tilde{K} \circ \mu(x, y)$ by $k_{\alpha}(x, y)$ and $K_{\alpha} \circ \mu(x, y)$, respectively.

Proof of Theorem 2.6. The proof of Theorem 2.5 applies with $K_{\alpha} \circ \mu(x, y)$ replaced by $K_{\alpha, p} \circ \mu(x, y)$.

Acknowledgements. The author thanks the referee for valuable comments that improved the presentation of the paper and for pointing out the reference [12].

\section{References}

[1] Adams, D. R., and L.-I. HedBerG: Function spaces and potential theory. - Grundlehren Math. Wiss. 314, Springer-Verlag, Berlin, 1996.

[2] Armitage D. H., and S. G. Gardiner: Classical potential theory. - Springer-Verlag, LondonBerlin-Heidelberg, 2001. 
[3] BJöRn, A, and J. BJöRn: Nonlinear potential theory on homogeneous spaces. - EMS Tracts Math. 17, Eur. Math. Soc., Zürich, 2011.

[4] Carleson L.: Selected problems on exceptional sets. - Mathematical Studies, Van Nostrand, Princeton, New Jersey, 1967.

[5] Coifman, R. R., and G. Weiss: Analyse harmonique non-commutative sur certains espaces homogenes. - Lecture Notes in Math. 242, Springer-Verlag, 1971.

[6] Deng, D., and Y. Han: Harmonic analysis on spaces of homogeneous type. - Lecture Notes in Math. 1966, Springer-Verlag, 2009.

[7] Deny, J. Sur les infinis d'un potentiel. - C. R. Acad. Sci. Paris 224, 1947, 524-525.

[8] Dоов, J. L.: Classical potential theory and its probabilistic counterpart. - Springer-Verlag, 1984.

[9] Evans, G. C.: On potentials of positive mass. Part II. - Trans. Amer. Math. Soc. 38, 1935, 201-236.

[10] Fuglede, B.: On the theory of potentials in locally compact spaces. - Acta Math. 103, 1960, $139-215$.

[11] Frostman, O.: Potentiel d'équilibre et capacité des ensembles avec quelques applications à la théorie des fonctions. - Thèse, Lund, 1935.

[12] Hajibayov, M. G.: Continuity properties of potentials on spaces of homogeneous type. - Int. J. Math. Anal. 2, 2008, 315-328.

[13] Havinson, S. JA.: A universal potential for compact sets of capacity zero, and other approximation characteristics of sparse sets. - Izw. Akad. Nauk Armjan. SSR Ser. Mat. 10:4, 1975, 356-372 (in Russian).

[14] Jonsson, A., and H. Wallin: Function spaces on subsets of $\mathbf{R}^{n}$. - Mathematical Reports, Gordon and Breach, Amsterdam, 1984.

[15] KellogG, O. D.: Foundations of potential theory. - Grundlehren Math. Wiss. 31, SpringerVerlag, Berlin-Heidelberg-New York, 1967.

[16] Kinnunen, J., R. Korte, T. Kuusi, M. Parvianen: Nonlinear parabolic capacity and polar sets of superparabolic functions. - Math. Ann. 355, 2013, 1349-1381.

[17] Landkof, N. S.: Foundations of modern potential theory. - Grundlehren Math. Wiss. 180, Springer-Verlag, Berlin-Heidelberg-New York, 1972.

[18] Macias, R. A., and C. Segovia: Lipschitz functions on spaces of homogeneous type. - Adv. Math. 33, 1979, 257-270.

[19] Simmons, G. F.: Introduction to topology and modern analysis. International student edition. - McGraw-Hill, 1963.

[20] SJödin, T: Bessel potentials and extension of continuous functions on compact sets. - Ark. Mat. 13:2, 1974, 263-271.

[21] SJöDIn, T.: A note on capacity and Hausdorff measure in homogeneous spaces. - Pot. Anal. $6: 1,1997,87-97$.

[22] Wallin, H.: Continuous functions and potential theory. - Ark. Mat. 5:1, 1963, 55-84. 